of science, the association of the maker's name with every article of equipment mentioned tends to convey the impression that he is looking through a well-written catalogue; but it must be remembered that this publication is primarily a trade journal and that makers' names are invaluable to those who may wish to purchase plant similar in nature to that described. In addition to the main articles, a number of short notes are included. These consist of personal news, information regarding new companies, company reports, book reviews, deseriptions of new equipment and miscellaneous notes. The whole appearance of the journal is attractive, and those whose time is not yet fully occupied with other publications of a similar nature may well be tempted to look through its pages.

\section{World Trade Digest}

MuCH useful information about industrial developments will be found in World Trade Digest, which commenced publication last November and is being issued monthly (from 45 Dorset Street, London, W.1. ; 1s.). The articles, which are well condensed and informative, are drawn from a wide range of periodicals, many of which are of a specialist nature and therefore unlikely to be readily available to the general reader. The first issue indicates that the editor will cast his net wide, for it covers such diverse subjects as the zip fastener, the channel tunnel, automatic irrigation, control of plant growth and corrosion resistance. An article on air freight, by $\mathbf{A}$. James Payne, condensed from the Recorder, refers to the million tons of air freight carried since the War and explains that air transport saves time, reduces risks of pilferage and damage in transit, and cuts down costs on crating and packaging. British aircharter companies are now regularly carrying such things as samples, penicillin, early fruit and flowers, machinery parts for urgent repair jobs, pedigree cattle, radio sets and delicate scientific instruments. Recently, an Air-brokers Association was formed from members of the Baltic Exchange, and this should prove to be a great step forward in regularizing air-freight business.

\section{British Standard for Photographic Lenses}

BrIrrsh Standard 1019 (1949) is the revised form of a 1942 war-emergency standard. It is the first of a series of British Standards for lenses and is essentially in agreement with several United States equivalents. The Standard, which is divded into three sections and two appendixes, applies to camera and enlarger lenses, with the exception of boxcamera lenses and single-component lenses in folding cameras. In the first section, definitions of the parts of a photographic lens, the focal lengths and associated quantities, and the lens aperture and associated quantities are given. The method of marking the focal length and the maximum aperture ratio on a photographic lens mount, together with the accuracy required for such markings, are specified in the second section. In this section, also, the standard series of stop sizes and the marking of distance scales are discussed. It is recommended in the third section that, in order to change to a stop of smaller aperturevalue, the iris of the lens should be rotated in an anti-clockwise direction as viewed from the front of the lens. The methods to be used for the measurement of focal lengths and of aperture and aperture ratio are fully described in the appendixes.
This Standard has been criticized for its lack of clarity and conciseness. It does, indeed, show signs of hasty, if not careless, compilation. In several places, in order to make the text clear to the reader, textual notes have been added, but they have been inserted in the wrong places; for example, Note 2, which should follow $3 g$, follows $4 c$. Terms, such as the lens axis and nodal point, are used without definition, and in one method described the user of the Standard is expected to have previous knowledge of the position of the axial point. For methods for determining the latter point, he is referred to an out-of -theway report by M. Hotine on air survey cameras. The Standard, in spite of its faults, fills a definite need, and it is to be hoped that an improved version will as soon as possible replace the present issue.

\section{Distribution of Population in England and Wales}

Is our note on the Registrar General's estimates of the local civilian population, which appeared in Nature of December 24, 1949, p. 1078, reference was made to the exclusion of conscripts away on national service from the local populations. The Registrar General for England and Wales has informed us that no information relating to the area of permanent residence of national service men is available. The number of conscripts on national service over the country as a whole could be obtained by increasing the figure for males aged 18-24 by about 44 per cent. It would, however, be unsafe to apply this ratio to local populations, as the distribution of industries varies and as there is differential reservation in different industries. In the estimate of local population for mid-1949, which is soon to be published, the number of men in the armed forces stationed in each area will be included. The exclusion of national service men from the local figures may be unavoidable; but we still think it unfortunate. For most purposes for which local population figures are required, it would be better to include national service men, as their absence is only of comparatively short duration. Indeed, the figure of conscripts is much more relevant to planners than an estimate of the number of men stationed in the area. The Service authorities should be able to provide the Registrar General with the material for obtaining at least an estimate of the conscripts from each area, which could be published separately from the main figures.

\section{Use of Film in a Research Establishment}

As article by J. Rogers in the second Bulletin of the Scientific Film Association describes the use of films in research establishments. These include its use as a research tool, as a means of recording experimental work and of explaining and making known the results of such work ; it may also be used for keeping the staff informed of the work of others. With these objects in view, a film unit was set up at the Building Research Station in 1947. So far its main use has been as a medium of explanation and information, although it has also been used for research and record purposes. High-speed cinematography has been used in research on flooring materials and has yielded interesting information on the action of an abrasion testing machine. Films are being used for record purposes in the testing of building materials to destruction, thus providing a means of re-witnessing tests which would be difficult and costly to repeat; film records are also made of certain building operations which are the subject of 